\title{
Miliary lung metastases from ROS1-rearranged lung adenocarcinoma: A case report
}

\author{
RYOTA OTOSHI $^{1}$, AKIMASA SEKINE ${ }^{1}$, KOJI OKUDELA ${ }^{2}$, TAKUMA KATANO $^{1}$, MASATO ASAOKA $^{1}$, \\ SATOSHI IKEDA ${ }^{1}$, TOMOHISA BABA ${ }^{1}$, SHIGERU KOMATSU ${ }^{1}$, ERI HAGIWARA ${ }^{1}$ and TAKASHI OGURA ${ }^{1}$ \\ ${ }^{1}$ Department of Respiratory Medicine, Kanagawa Cardiovascular and Respiratory Center, Yokohama, Kanagawa 236-0051; \\ ${ }^{2}$ Department of Pathology, Yokohama City University Graduate School of Medicine, Yokohama, Kanagawa 236-0027, Japan
}

Received May 28, 2019; Accepted April 6, 2020

DOI: $10.3892 / \mathrm{mco} .2020 .2040$

\begin{abstract}
Miliary lung metastases are a rare form of metastasis of non-small-cell lung carcinoma. Miliary lung metastases commonly develop in lung adenocarcinoma with epidermal growth factor receptor mutation. In the present study, we present a case of miliary lung metastases from lung adenocarcinoma with ROS1 rearrangement. The patient, who had a history of surgery for stage IIIA lung adenocarcinoma, presented to our hospital with cough, dyspnea, and severe hypoxia. Chest computed tomography showed numerous tiny, randomly distributed nodules throughout both lungs. No metastases were observed in other organs. Molecular profiling of the surgical specimens was positive for ROS1 rearrangement. The results suggest that chest physicians should be aware that miliary lung metastases can develop in patients with lung adenocarcinoma with ROS1 rearrangement.
\end{abstract}

\section{Introduction}

Miliary lung metastases are a rare form of metastasis, accounting for $1-2 \%$ of cases of non-small-cell lung carcinoma (NSCLC). Miliary metastases have been reported to be more commonly found in patients with NSCLCs with an epidermal growth factor receptor (EGFR) mutation $(1,2)$. However, there is only one case report on miliary lung metastases from NSCLC with ROS1 rearrangement (3). We report such a case in a patient with ROS1-rearranged lung adenocarcinoma.

\section{Case report}

A 50-year-old woman who had never smoked underwent right middle lobectomy for stage IIIA lung micropapillary

Correspondence to: Dr Ryota Otoshi, Department of Respiratory Medicine, Kanagawa Cardiovascular and Respiratory Center, 6-16-1 Tomioka-higashi, Yokohama, Kanagawa 236-0051, Japan

E-mail: ootoshi@kanagawa-junko.jp

Key words: ROS1, miliary metastases, adenocarcinoma, non-small cell adenocarcinoma in June 2017. She refused adjuvant chemotherapeutic treatment. In February 2018, the follow-up computed tomography (CT) did not reveal any evidence of recurrence. The patient discontinued hospital visit on her own judgment. She presented to Kanagawa Cardiovascular and Respiratory Center (Yokohama, Japan) in November 2018 complaining of a 1-month history of cough and dyspnea. She had severe hypoxia with a pulse oximetry reading of $85 \%$ on room air; thus, oxygen therapy at $5 \mathrm{l} / \mathrm{min}$ was started. Chest CT showed numerous tiny, randomly distributed nodules throughout both lungs, bilateral patchy consolidation, mediastinal lymphadenopathy, and intralobular septal thickening (Fig. 1). No metastases were observed in other organs. Laboratory examinations revealed carcinoembryonic antigen levels to be $84.6 \mathrm{ng} / \mathrm{ml}$ (normal, $<5.0 \mathrm{ng} / \mathrm{ml}$ ), KL-6 level to be $3,731 \mathrm{U} / \mathrm{ml}$ (normal, $<500 \mathrm{U} / \mathrm{ml}$ ), and lactase dehydrogenase (LDH) level to be $1,859 \mathrm{U} / 1$ (normal, <222 U/1). Based on these results, a diagnosis of recurrent lung cancer with miliary lung metastases and lymphangiosis carcinomatosa was made.

Molecular analysis of the surgical specimens revealed no EGFR mutation or anaplastic lymphoma kinase (ALK) rearrangement, but there was ROS1 rearrangement as determined using reverse transcription polymerase chain reaction using primer sequences according to the manufacturer's instructions of the ROS1 fusion gene detection kit (Amoy Diagnostics Co., Ltd.). Treatment with crizotinib (200 mg/day orally) was administered. However, after 5 days of treatment, the patient's respiratory condition rapidly worsened, requiring nasal high-flow oxygen with a fraction of inspired oxygen of $90 \%$. Chest CT revealed an increase in the number of miliary nodules and worsening of consolidation (Fig. 2). The LDH level increased to $4503 \mathrm{U} / 1$. However, we could not determine whether the aggravation of her respiratory condition was due to drug-induced pneumonitis or disease progression because her respiratory condition rapidly worsened and could not be assessed by biopsy due to high risk of further deterioration. Crizotinib treatment was discontinued and pulse steroids were administered. However, the patient's condition failed to improve, and her performance status score fell to 2. Therefore, she was started on a therapy with cisplatin, pemetrexed, and bevacizumab and closely observed.

After the first cycle of chemotherapy, CT showed regression of the nodules and consolidation. Her respiratory condition dramatically improved, and she was treated with 

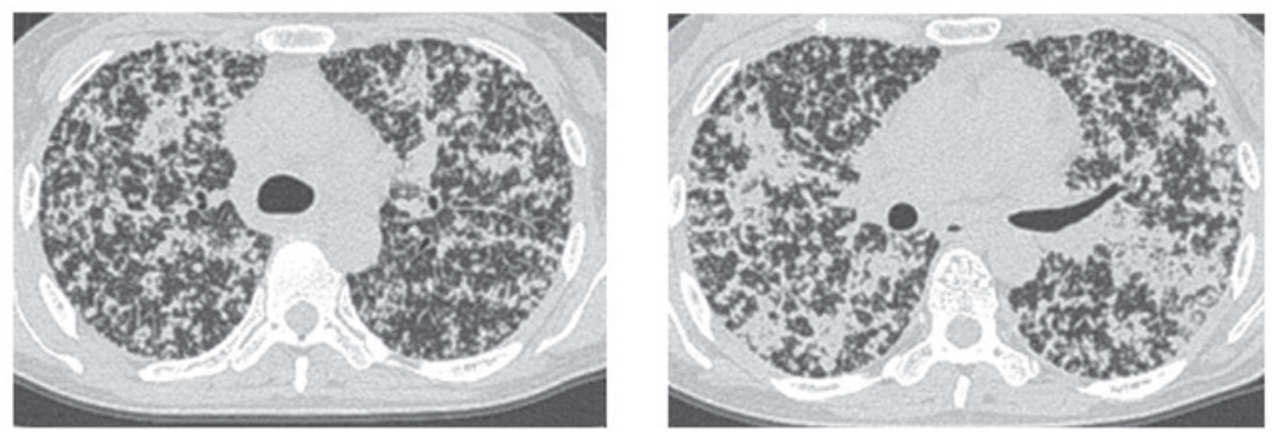

Figure 1. Chest computed tomography from admission showing numerous tiny nodules and patchy consolidation throughout both lungs, mediastinal lymphadenopathy, and intralobular septal thickening.
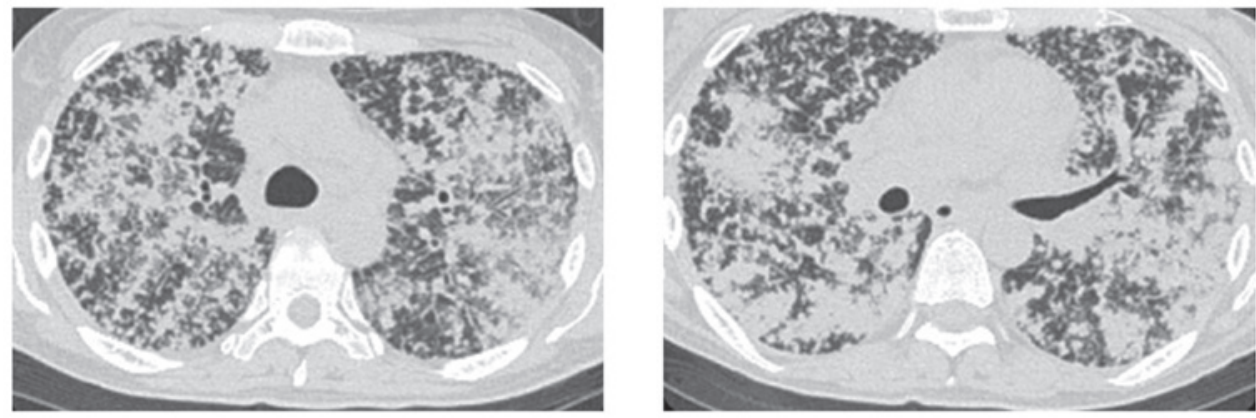

Figure 2. Computed tomography after treatment with crizotinib shows worsening of consolidation and ground-glass attenuation.
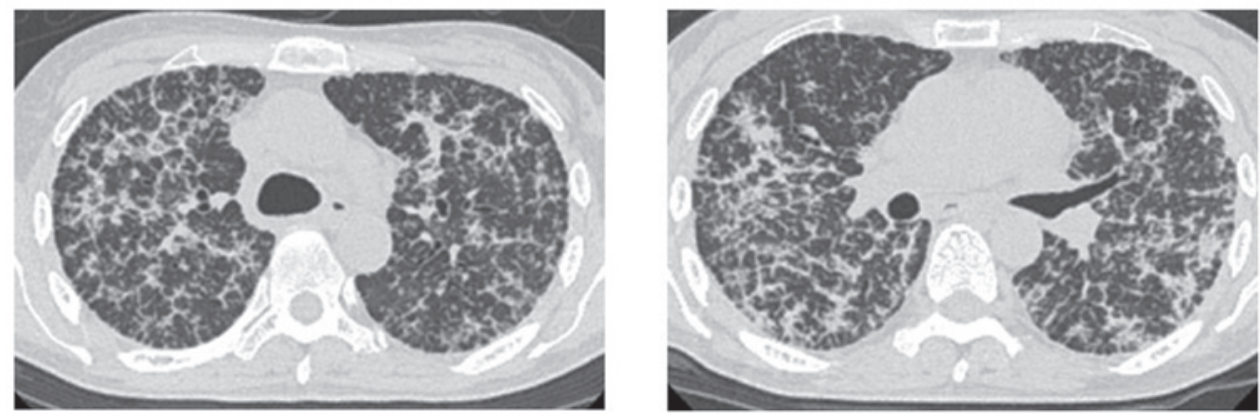

Figure 3. Computed tomography showing improvement of the miliary metastases after the first cycle of cisplatin, pemetrexed, and bevacizumab.

only 1 1/min of nasal oxygen (Fig. 3). At the same time, her CEA and LDH levels decreased to $42.7 \mathrm{ng} / \mathrm{ml}$ and 2,755 U/1, respectively. In May 2019, she received maintenance therapy with pemetrexed and bevacizumab following four cycles of platinum-based chemotherapy and continued five cycles of maintenance therapy until the deterioration of lung metastases.

\section{Discussion}

In the present study, we report a case of miliary lung metastases from ROS1-rearranged lung adenocarcinoma. Miliary lung metastases have been described as a rare form of metastases observed in 1-2\% of cases of NSCLC $(1,2)$. These cases are reported to occur more commonly in association with an EGFR mutation. Laack et al reported five cases of miliary lung metastases in NSCLCs with exon 19 deletion (4). Hsu et al reported that patients with NSCLC with an EGFR mutation were three times more likely to present with miliary metastases than those without the mutation, indicating the importance of evaluating the status of EGFR mutation in cases of NSCLC with miliary lung metastases (5). On the other hand, miliary lung metastases from NSCLC have also been reported with a different oncogenic driver mutation. Falk et al reported miliary lung metastases from NSCLC with an ALK rearrangement (6). With regard to ROS1-mutated NSCLC, we found only one case report of miliary lung metastases: This patient was successfully treated by crizotinib (3). By contrast, our patient was markedly improved by combination cytotoxic chemotherapy consisting of cisplatin, pemetrexed, and bevacizumab after discontinuing crizotinib treatment. Our case as well as those cited above indicate that oncogenic driver mutations may be correlated with miliary lung metastases.

ROS1 rearrangement is considered a rare oncogenic mutation, reported in only $1-2 \%$ of cases of NSCLC (7). Chest 
physicians should be aware that miliary lung metastases can develop in patients with lung adenocarcinoma with oncogenic driver mutations other than an EGFR mutation, including ROS1 rearrangement. However, crizotinib did not have a desired effect in our case, whereas one reported case of miliary lung metastases from ROS1-rearranged lung adenocarcinoma was successfully treated by crizotinib (3). Although the reason for crizotinib being ineffective in our case remains unclear, the objective response rate of crizotinib for ROS1-rearranged NSCLC patients is reportedly 69.3-72\%; about $30 \%$ of them did not respond to crizotinib adequately, as seen in our case $(8,9)$.

In conclusion, we present a case of miliary lung metastases from a ROS1-rearranged lung adenocarcinoma. Chest physicians should be aware that miliary lung metastases can occur in patients with NSCLCs with oncogenic driver mutations other than an EGFR mutation, including ROS1 rearrangement. We await reports of additional cases similar to ours.

\section{Acknowledgements}

Not applicable.

\section{Funding}

No funding was received.

\section{Availability of data and materials}

The datasets used and/or analyzed during the present study are available from the corresponding author on reasonable request.

\section{Authors' contributions}

RO and AS analyzed and interpreted the data and wrote the manuscript. RO, AS, TK, MA, SI, TB, SK, EH and TO evaluated the patient and participated in the therapy. KO evaluated the pathological specimens. All authors have read and approved the final manuscript.

\section{Ethics approval and consent to participate}

Not applicable.

\section{Patient consent for publication}

The patient provided written informed consent for the publication of the case details and any associated images.

\section{Competing interests}

Although Dr Sekine and Dr Ikeda have received lecture fees from Chugai Pharmaceuticals, there was no drug supply and funding for this study. All authors have stated that they have no conflicts of interest related to this article to disclose.

\section{References}

1. Umeki S: Association of miliary lung metastases and bone metastases in bronchogenic carcinoma. Chest 104: 948-950, 1993.

2. Wu SG, Hu FC, Chang YL, Lee YC, Yu CJ, Chang YC, Wu JY, Shih JY and Yang PC: Frequent EGFR mutations in nonsmall cell lung cancer presenting with miliary intrapulmonary carcinomatosis. Eur Respir J 41: 417-424, 2013.

3. Brindel A, Huet D, Vaillant P, Vignaud JM and Tiotiu A: ROS-1 rearranged bronchopulmonary adenocarcinoma revealed by a pulmonary miliary. Bull Cancer 105: 549-551, 2018.

4. Laack E, Simon R, Regier M, Andritzky B, Tennstedt P, Habermann C, Verth CZ, Thöm I, Grob T, Sauter G and Bokemeyer C: Miliary never-smoking adenocarcinoma of the lung: Strong association with epidermal growth factor receptor exon 19 deletion. J Thorac Oncol 6: 199-202, 2011.

5. Hsu F, Nichol A, Toriumi T and De Caluwe A: Miliary metastases are associated with epidermal growth factor receptor mutations in non-small cell lung cancer: A population-based study. Acta Oncol 56: 1175-1180, 2017.

6. Falk AT, Poudenx M, Otto J, Ghalloussi H and Barrière J: Adenocarcinoma of the lung with miliary brain and pulmonary metastases with echinoderm microtubule-associated protein like 4-anaplastic lymphoma kinase translocation treated with crizotinib: A case report. Lung Cancer 8: 282-284, 2012.

7. Rothschild SI and Gautschi O: Crizotinib in the treatment of non-small-cell lung cancer. Clin Lung Cancer 4: 473-480, 2013.

8. Shaw AT, Ou SH, Bang YJ, Camidge DR, Solomon BJ, Salgia R, Riely GJ, Varella-Garcia M, Shapiro GI, Costa DB, et al: Crizotinib in ROS1-rearranged non-small-cell lung cancer. N Engl J Med 371: 1963-1971, 2014.

9. Wu YL, Yang JC, Kim DW, Lu S, Zhou J, Seto T, Yang JJ, Yamamoto N, Ahn MJ, Takahashi T, et al: Phase II study of crizotinib in east asian patients with ROS1-positive advanced non-small-cell lung cancer. J Clin Oncol 36: 1405-1411, 2018. 